Publ. Math. Debrecen

Manuscript (January 09, 2013)

\title{
On the number of solutions of binomial Thue inequalities
}

\author{
By M. A. Bennett, I. Pink and Zs. Rábai
}

\begin{abstract}
Let $a, b$ and $n$ be positive integers with $n \geq 3$ and consider the binomial Thue inequality $\left|a x^{n}-b y^{n}\right| \leq 3$. In this paper, we extend a result of the first author [10] and prove that, apart from finitely many explicitly given exceptions, this inequality has at most a single solution in positive integers $x$ and $y$. In the proof, we combine lower bounds for linear forms in logarithms of algebraic numbers with the hypergeometric method of Thue-Siegel and an assortment of techniques from computational Diophantine approximation.
\end{abstract}

\section{Introduction}

A classical problem in number theory is the approximation of algebraic numbers by rationals, underlying which one has a theorem of Liouville :

Theorem 1.1. (Liouville, 1844) If $\alpha$ is a given algebraic number of degree $n \geq 2$, then there exists an effectively computable constant $c(\alpha)$ such that, for every $\frac{x}{y} \in \mathbb{Q}$ with $y>0$, we have

$$
\left|\alpha-\frac{x}{y}\right|>\frac{c(\alpha)}{y^{n}}
$$

Mathematics Subject Classification: 11D41, 11D61.

Key words and phrases: Thue equations, diophantine equations.

The research was supported in part by NSERC (M. A. B.), by the TÁMOP 4.2.1./B-09/1/KONV-2010-0007 project, and grant K75566 of the Hungarian National Foundation for Scientific Research (I. P.), by the Hungarian Academy of Sciences, and the TÁMOP 4.2.1./B-09/1/KONV-2010-0007, TÁMOP-4.2.2/B-10/1-2010-0024 projects, and the TÁMOP 4.2.4. A/2-11-1-2012-0001 „National Excellence Program - Elaborating and operating an inland student and researcher personal support system" (Zs. R.). The TÁMOP projects were subsidized by the European Union and co-financed by the European Social Fund and the European Regional Development Fund. 
For applications to Diophantine equations, it is of utmost importance to reduce the exponent $n$ here, i.e. to deduce like inequalities with some exponent $\lambda<n$. In full generality, the first such result was due to Thue [38] who proved the following theorem.

Theorem 1.2. (Thue, 1909) If $\alpha$ is an algebraic number of degree $n \geq 3$, then, given $\varepsilon>0$, there exists an effectively computable constant $c(\alpha, \varepsilon)$ such that for all integers $x$ and $y>0$ we have

$$
\left|\alpha-\frac{x}{y}\right|>\frac{c(\alpha, \varepsilon)}{y^{\frac{n}{2}+1+\varepsilon}} .
$$

From this result, Thue deduced that if $F(x, y) \in \mathbb{Z}[x, y]$ is an irreducible binary form of degree $n \geq 3$, and $m$ is a fixed nonzero integer then the corresponding Thue equation

$$
F(x, y)=m
$$

has at most finitely many solutions in integers $x$ and $y$. This result is, however, ineffective in the sense that it does not provide any way to actually compute $c(\alpha, \varepsilon)$, and hence cannot be applied to determine the solutions of the corresponding equations.

Whilst there is now a well-developed literature on effective solution of Thue equations, based upon a variety of techniques (including, for instance, lower bounds for linear forms in logarithms of algebraic numbers; see e.g. [4]), in the paper at hand, we will concentrate on bounding the number of solutions to such equations, rather than their heights. In this regard, it is known that the number of solutions to equation (1.1) in integers is bounded above in terms of only the degree of $F$ and the number of distinct prime divisors of $m$ (see e.g. Bombieri and Schmidt [19]). We will restrict our attention to what is, in some sense, the simplest possible case, that of binomial Thue equations and inequalities. For these equations, the number of such solutions is bounded in terms of $m$ alone (see Mueller and Schmidt [33]). Despite the fact that the situation we will consider is a very specialized one, we believe it is instructive to see what can be said explicitly, as a test of the current state of refinement of computational and analytic techniques. As a starting point, we note that, implicit in the techniques of [10] and [16] is the following result.

Theorem 1.3. Let $c$ be a positive integer. Then there exists an effectively computable finite set $S_{c}$ of triples of positive integers $a, b$ and $n$ with the property that if $a, b$ and $n \geq 3$ are any positive integers for which the Diophantine inequality

$$
\left|a x^{n}-b y^{n}\right| \leq c
$$

has more than a single solution in positive integers $x$ and $y$, then $(a, b, n) \in S_{c}$. 
The main result of [10] is that the set $S_{1}$ is empty. In treating (1.2), we will have occasion to consider the corresponding equation

$$
\left|a x^{n}-b y^{n}\right|=c,
$$

where $a, b$ and $c$ are given positive integers, and $x, y$ and $n$ are unknown integers. Siegel [37], refining earlier work of Thue, showed that if the coefficients $a$ and $b$ are large enough compared to $c$ and $n$, then (1.3) has at most one positive solution. Later, Evertse [21] was able to substantially sharpen Siegel's theorem (see our Lemma 2.2). Both results depend on the so-called hypergeometric method. Related work in this area, including applications and generalizations to cases where $a$ and $b$ are taken to be $S$-units rather than fixed, may be found in, for example, Mahler [30], [31], Baker [1], [2], [3], Chudnovsky [20] and many, many other papers, including [5]. [6], [7], [8], [9], [10], [11], [14], [17], [18], [22], [23], [24], [25], [26], [32] and [39].

The main result of the paper at hand is the following.

Theorem 1.4. With $S_{c}$ defined as in the statement of Theorem 1.3, we have $S_{3} \subseteq$ $S_{3}^{*} \cup T_{3}$, where

$$
S_{3}^{*}=\{(1,2,3),(2,1,3),(1,3,3),(3,1,3),(2,5,3),(5,2,3)\}
$$

and

$$
T_{3}=\{(1,3, n),(3,1, n),(2,5, n),(5,2, n) \text { with } 37 \leq n \leq 347, n \text { prime }\}
$$

For $(a, b, n) \in S_{3}^{*}$, the solutions in positive integers to inequality (1.2) with $c=3$ are, in each case, $(x, y)=(1,1)$, and also

\begin{tabular}{c|c|c|c|c|c|c}
$(a, b, n)$ & $(1,2,3)$ & $(2,1,3)$ & $(1,3,3)$ & $(3,1,3)$ & $(2,5,3)$ & $(5,2,3)$ \\
\hline$(x, y)$ & $(5,4)$ & $(4,5)$ & $(3,2)$ & $(2,3)$ & $(19,14)$ & $(14,19)$
\end{tabular}

In case $n=3$, this theorem represents a slight sharpening of a classical result of Ljunggren [29], who considered equation (1.3) with $n=3$ and $c \in\{1,3\}$. It is very likely that $S_{3}=S_{3}^{*}$ (which should be provable with a finite but currently infeasible amount of computation). We can, in any case, certainly prove a sharpened version of Theorem 1.4, with $T_{3}$ replaced by a somewhat smaller set, through more careful application of the hypergeometric method; in our opinion the effort involved would somewhat exceed the payoff. 


\section{Some lemmata}

In this section, we collect a number of lemmata that we use in the proof of Theorem 1.4. The first is a state-of-the-art lower bound for linear forms in the logarithms of two algebraic numbers, due to Laurent (Theorem 2 of [28]). For any algebraic number $\alpha$ of degree $d$ over $\mathbb{Q}$, we define as usual the absolute logarithmic height of $\alpha$ by the formula

$$
h(\alpha)=\frac{1}{d}\left(\log \left|a_{0}\right|+\sum_{i=1}^{d} \log \max \left(1,\left|\alpha^{(i)}\right|\right)\right),
$$

where $a_{0}$ is the leading coefficient of the minimal polynomial of $\alpha$ over $\mathbb{Z}$ and the $\alpha^{(i)} \mathbf{s}$ are the conjugates of $\alpha$ in the field of complex numbers.

Lemma 2.1. Let $\alpha_{1}$ and $\alpha_{2}$ be multiplicatively independent algebraic numbers, $h$, $\rho$ and $\mu$ be real numbers with $\rho>1$ and $1 / 3 \leq \mu \leq 1$. Set

$$
\begin{gathered}
\sigma=\frac{1+2 \mu-\mu^{2}}{2}, \quad \lambda=\sigma \log \rho, \quad H=\frac{h}{\lambda}+\frac{1}{\sigma} \\
\omega=2\left(1+\sqrt{1+\frac{1}{4 H^{2}}}\right), \quad \theta=\sqrt{1+\frac{1}{4 H^{2}}}+\frac{1}{2 H} .
\end{gathered}
$$

Consider the linear form $\Lambda=b_{2} \log \alpha_{2}-b_{1} \log \alpha_{1}$, where $b_{1}$ and $b_{2}$ are positive integers. Put

$$
D=\left[\mathbb{Q}\left(\alpha_{1}, \alpha_{2}\right): \mathbb{Q}\right] /\left[\mathbb{R}\left(\alpha_{1}, \alpha_{2}\right): \mathbb{R}\right]
$$

and assume that

$$
\begin{gathered}
h \geq \max \left\{D\left(\log \left(\frac{b_{1}}{a_{2}}+\frac{b_{2}}{a_{1}}\right)+\log \lambda+1.75\right)+0.06, \lambda, \frac{D \log 2}{2}\right\}, \\
a_{i} \geq \max \left\{1, \rho\left|\log \alpha_{i}\right|-\log \left|\alpha_{i}\right|+2 D h\left(\alpha_{i}\right)\right\} \quad(i=1,2),
\end{gathered}
$$

and

$$
a_{1} a_{2} \geq \lambda^{2}
$$

Then

$$
\log |\Lambda| \geq-C\left(h+\frac{\lambda}{\sigma}\right)^{2} a_{1} a_{2}-\sqrt{\omega \theta}\left(h+\frac{\lambda}{\sigma}\right)-\log \left(C^{\prime}\left(h+\frac{\lambda}{\sigma}\right)^{2} a_{1} a_{2}\right)
$$

with

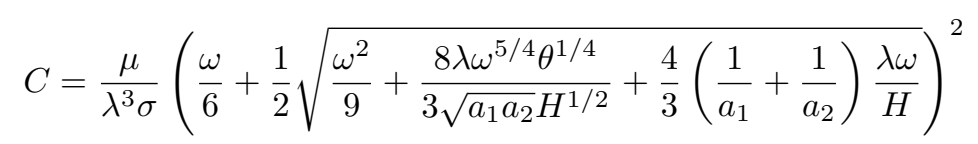

and

$$
C^{\prime}=\sqrt{\frac{C \sigma \omega \theta}{\lambda^{3} \mu}}
$$


The next lemma is a result of Evertse (Theorem 2.1 of [21]) and, as mentioned earlier, represents a refinement of prior work of Siegel on the hypergeometric method.

Lemma 2.2. Suppose that $a, b, c$ and $n$ are positive integers with $n \geq 3$. Define

$$
T_{n}=3^{-\frac{n-2}{n}} n \prod_{p \mid n} p^{\frac{1}{p-1}}, \quad \mu_{3}=T_{3}^{11 / 2}, \quad \mu_{n}=T_{n}^{\max \left\{\frac{n+2}{2(n-3)}, \frac{n}{n-2}\right\}} \text { if } n \geq 4
$$

and

$$
\alpha_{3}=9, \quad \alpha_{n}=\max \left\{\frac{3 n-2}{2(n-3)}, \frac{2(n-1)}{n-2}\right\} \text { if } n \geq 4 .
$$

Then the inequality (1.2) has at most one solution in positive coprime integers $x$ and $y$ satisfying

$$
\max \left\{a x^{n}, b y^{n}\right\} \geq \mu_{n} c^{\alpha_{n}} .
$$

The final three lemmata we will use are results of the first author [8], [9], [10] and [13]. To be precise, they are a combination of Theorem 5.2 of [10] with Theorem 5.2 of [13], a special case of Theorem 1.1 of [8], and a special case of Theorem 1.1 of [9], respectively. We will use them to treat inequality (1.2) for "small" values of $n$.

Lemma 2.3. Suppose $b>a$ are coprime positive integers and $m=\left[\frac{n+1}{3}\right]$. Let $n$, $c_{1}(n)$ and $d(n)$ be as given in the following table. 


\begin{tabular}{|ccc|ccc|ccc|}
\hline$n$ & $c_{1}(n)$ & $d(n)$ & $n$ & $c_{1}(n)$ & $d(n)$ & $n$ & $c_{1}(n)$ & $d(n)$ \\
\hline 17 & 8.93 & 13.06 & 107 & 83.55 & 50.84 & 227 & 201.15 & 116.91 \\
19 & 9.40 & 15.46 & 109 & 84.18 & 58.97 & 229 & 202.11 & 100.61 \\
23 & 13.03 & 17.66 & 113 & 89.22 & 77.93 & 233 & 207.50 & 102.49 \\
29 & 17.39 & 29.95 & 127 & 100.47 & 72.61 & 239 & 213.74 & 105.66 \\
31 & 17.92 & 30.55 & 131 & 105.34 & 71.51 & 241 & 214.95 & 95.14 \\
37 & 21.2 & - & 137 & 111.44 & 79.94 & 251 & 226.83 & 115.64 \\
41 & 25.83 & 36.08 & 139 & 112.15 & 77.27 & 257 & 233.75 & 113.23 \\
43 & 26.62 & 33.95 & 149 & 122.53 & 85.82 & 263 & 240.15 & 119.49 \\
47 & 30.46 & 40.16 & 151 & 123.41 & 89.04 & 269 & 246.54 & 124.75 \\
53 & 34.78 & 35.37 & 157 & 129.07 & 81.61 & 271 & 247.72 & 134.21 \\
59 & 39.18 & 48.34 & 163 & 134.80 & 93.64 & 277 & 254.62 & 119.17 \\
61 & 39.96 & 55.93 & 167 & 139.95 & 82.87 & 281 & 260.46 & 116.79 \\
67 & 44.76 & 43.56 & 173 & 146.07 & 87.71 & 283 & 261.67 & 118.21 \\
71 & 48.36 & 54.80 & 179 & 151.40 & 83.92 & 293 & 274.23 & 129.73 \\
73 & 52.83 & 48.11 & 181 & 152.20 & 91.69 & 307 & 289.00 & 124.89 \\
79 & 58.27 & 54.65 & 191 & 163.78 & 84.40 & 311 & 294.70 & 130.14 \\
83 & 62.70 & 49.64 & 193 & 164.81 & 91.51 & 313 & 296.38 & 130.18 \\
89 & 67.56 & 60.29 & 197 & 170.17 & 104.53 & 317 & 302.73 & 134.63 \\
97 & 73.71 & 62.14 & 199 & 170.80 & 110.41 & 331 & 317.41 & 147.69 \\
101 & 78.29 & 50.36 & 211 & 183.12 & 124.02 & 337 & 324.63 & 139.95 \\
103 & 79.16 & 60.85 & 223 & 195.74 & 112.93 & 347 & 338.02 & 133.98 \\
\hline
\end{tabular}

If

$$
(\sqrt[m]{b}-\sqrt[m]{a})^{m} e^{c_{1}(n)}<1,
$$

then, for all $x$ and $y>0$ integers, we have

$$
\left|\left(\frac{b}{a}\right)^{1 / n}-\frac{x}{y}\right|>\left(C_{2}(\sqrt[m]{b}+\sqrt[m]{a})^{m}\right)^{-1} y^{-\lambda_{1}}
$$

where

$$
C_{2}=\left\{\begin{array}{cc}
3.15 \cdot 10^{24}(m-1)^{2} n^{m-1} e^{c_{1}(n)+d(n)} & \text { if } n \neq 37 \\
5 \cdot 10^{75} & \text { if } n=37
\end{array}\right. \text {, }
$$

and

$$
\lambda_{1}=(m-1)\left\{1-\frac{\log \left((\sqrt[m]{b}+\sqrt[m]{a})^{m} e^{c_{1}(n)+1 / 20}\right)}{\log \left((\sqrt[m]{b}-\sqrt[m]{a})^{m} e^{c_{1}(n)}\right)}\right\} .
$$


Lemma 2.4. Let $c \in\{1,2,3\}$ and a be a positive integer which satisfies

$$
8(\sqrt{a}+\sqrt{a+c})^{2}>c^{4} \cdot(\kappa(c))^{3},
$$

where

$$
\kappa(c)= \begin{cases}3 \sqrt{3} & \text { for } c=1,2 \\ \sqrt{3} & \text { for } c=3\end{cases}
$$

Then, for all positive integers $x$ and $y$,

$$
\left|\sqrt[3]{1+\frac{c}{a}}-\frac{x}{y}\right|>(4 \cdot a \cdot \kappa(c))^{-1}\left(10^{4} y\right)^{-\lambda_{3}},
$$

where

$$
\lambda_{3}=1+\frac{\log \left(\frac{\kappa(c)}{2}(\sqrt{a}+\sqrt{a+c})^{2}\right)}{\log \left(\frac{2}{c^{2} \cdot \kappa(c)}(\sqrt{a}+\sqrt{a+c})^{2}\right)} .
$$

Lemma 2.5. Let $a$ be a positive integer, $c \in\{1,2,3\}$ and $n \in\{4,5,7,11,13\}$. If

$$
(\sqrt{a}+\sqrt{a+c})^{2(n-2)}>c^{2(n-1)}\left(\frac{\kappa(c, n)}{c_{2}(n)}\right)^{n}
$$

then for all positive integers $x$ and $y$,

$$
\left|\sqrt[n]{1+\frac{c}{a}}-\frac{x}{y}\right|>\frac{1}{a} \cdot\left(10^{10} y\right)^{-\lambda_{4}}
$$

where

$$
\begin{gathered}
\lambda_{4}=1+\frac{\log \left(\frac{\kappa(c, n)}{c_{2}(n)}(\sqrt{a}+\sqrt{a+c})^{2}\right)}{\log \left(\frac{c_{2}(n)}{c^{2} \kappa(c, n)}(\sqrt{a}+\sqrt{a+c})^{2}\right)}, \quad \kappa(c, n)=\prod_{p \mid n} p^{\max \left\{\operatorname{ord}_{p}\left(\frac{n}{c}\right)+\frac{1}{p-1}, 0\right\}}, \\
c_{2}(4)=1.62, c_{2}(5)=1.84, c_{2}(7)=1.76, c_{2}(11)=1.67 \text { and } c_{2}(13)=1.65 .
\end{gathered}
$$

\section{Proof of Theorem 1.4}

We will consider the inequality

$$
\left|a x^{n}-b y^{n}\right| \leq 3
$$


in integer unknowns $x, y, a, b$ and $n$ which satisfy, without loss of generality,

$$
b>a \geq 1, n \geq 3, x \geq 1, y \geq 1 .
$$

We may further assume, again without loss of generality, that in (3.1) the exponent $n$ is either 4 or an odd prime. By Lemma 2.2, it follows that if

$$
x^{n} \geq \mu_{n} \cdot 3^{\alpha_{n}},
$$

then (3.1) has at most one solution in positive integers $x$ and $y$. This implies that, apart from when $n \in\{3,4,5\}$, inequality (3.1) has at most one positive solution with $x \geq 2$. We may thus distinguish two cases.

Case I: The inequality $(3.1)$ has $(x, y)=(1,1)$ as a solution. We thus have $b=a+c$ for $c \in\{1,2,3\}$ and hence are led to consider the inequality

$$
\left|a x^{n}-(a+c) y^{n}\right| \leq 3,
$$

where $c \in\{1,2,3\}$ and $a, x, y$ and $n$ are positive integers with $n \geq 3$.

Case II : We have $n \in\{3,4,5\}, b-a>3$ and inequality (3.1) has a solution in positive integers $x$ and $y$ with $x \geq 2$.

We first deal with Case I.

3.1. Linear forms in two logarithms. The main purpose of this subsection is to prove the following.

Theorem 3.1. If there is a solution to inequality (3.3) in positive integers $x$ and $y$ with $(x, y) \neq(1,1)$, then $n \leq 347$.

To prove this, we will have use of the following technical lemma.

Lemma 3.2. If inequality (3.3) has a solution in positive integers $(x, y) \neq(1,1)$ then $x>\frac{n a}{c}$.

Proof of Lemma 3.2 : If $x \leq y$ and $y>1$, then

$$
\left|a x^{n}-(a+c) y^{n}\right| \geq c y^{n}>3,
$$

contradicting (3.3). We may thus suppose that $x \geq y+1$, which by (3.3) yields

$$
a x^{n}-(a+c) y^{n} \geq a(y+1)^{n}-(a+c) y^{n} .
$$


By the binomial theorem, the right hand side of this is

$$
n a y^{n-1}+a\left(\left(\begin{array}{l}
n \\
2
\end{array}\right) y^{n-2}+\cdots+\left(\begin{array}{c}
n \\
n-1
\end{array}\right) y+1\right)-c y^{n} .
$$

Since

$$
a\left(\left(\begin{array}{l}
n \\
2
\end{array}\right) y^{n-2}+\cdots+\left(\begin{array}{c}
n \\
n-1
\end{array}\right) y+1\right)>3
$$

it follows from (3.3) that

$$
n a y^{n-1}-c y^{n}<0,
$$

which in turn implies that $x>y>\frac{n a}{c}$.

Proof of Theorem 3.1 Suppose that inequality (3.3) has a positive solution $(x, y) \neq$ $(1,1)$ with $n>347$. By Lemma 3.2, it follows that $x>n a / c$. We consider the linear form

$$
|\Lambda|=\left|\log \left(1+\frac{c}{a}\right)-n \log \left(\frac{x}{y}\right)\right| .
$$

Since (3.3) is equivalent to the inequality

$$
\left|1-\left(1+\frac{c}{a}\right)\left(\frac{y}{x}\right)^{n}\right| \leq \frac{3}{a x^{n}},
$$

and since, for every $z \in \mathbb{C}$ with $|z-1|<0.795$, we have $|\log (z)|<2|z-1|$, it follows that

$$
|\Lambda|<\frac{6}{x^{n}}
$$

We write

$$
\begin{gathered}
\alpha_{1}=\frac{x}{y}, \alpha_{2}=1+\frac{c}{a}, b_{1}=n, b_{2}=1, \mu=0.63, \sigma=0.93155, D=1, \\
\rho=1+\frac{\log (a+c)}{\log \left(1+\frac{c}{a}\right)}, \text { and choose } a_{1}=2.003 \log (x) \text { and } a_{2}=3 \log (a+c) .
\end{gathered}
$$

Applying Lemma 2.1, one may readily check that (2.3) holds. We distinguish two cases according to whether $a \geq 14$ or $a \leq 13$, respectively.

If $a \geq 14$ then, by calculus, we find that there exist absolute constants $c_{1}, c_{2}$ such that

$$
c_{1} \sigma \log (a+c)<\lambda<c_{2} \sigma \log (a+c)
$$


Here we may choose $c_{2}=1.3646$ if $c=1, c_{2}=1.1835$ if $c=2$ and $c_{2}=1.1226$ if $c=$ 3. The corresponding values of $c_{1}$ are $c_{1}=1$ if $c \in\{1,2\},\left(c_{1}, a\right)=(0.96,14),(0.98,16)$, or $(0.99,17)$, if $c=3$ and $14 \leq a \leq 17$, and $c_{1}=1$ if $c=3$ and $a \geq 18$. Since $n>347$ and $x>\frac{n a}{c}$, it follows that $\frac{\log (a+c)}{\log (x)}<1$ and, via $(3.7)$,

$\log \left(\frac{n}{3 \log (a+c)}+\frac{1}{2.003 \log (x)}\right)+\log (\lambda)+1.81<\log \left(\frac{c_{2} \sigma n}{3}+\frac{c_{2} \sigma}{2.003}\right)+1.81$

Hence, for $a \geq 14$, we may take

$$
h=\max \left\{\log \left(\frac{c_{2} \sigma n}{3}+\frac{c_{2} \sigma}{2.003}\right)+1.81, \lambda\right\} .
$$

Suppose first that $h=\log \left(\frac{c_{2} \sigma n}{3}+\frac{c_{2} \sigma}{2.003}\right)+1.81$. Then, by (3.7) and the assumption that $a \geq 14$,

$$
\frac{h}{\lambda}+\frac{1}{\sigma} \leq A:=\frac{\log \left(\frac{c_{2} \sigma n}{3}+\frac{c_{2} \sigma}{2.003}\right)+1.81}{\sigma c_{1} \log (a+c)}+\frac{1}{\sigma} .
$$

Lemma 2.1 and (3.8) together imply that

$$
\log |\Lambda|>-C \lambda^{2} a_{1} a_{2} A^{2}-\sqrt{\omega \theta} \lambda A-\log \left(C^{\prime} a_{1} a_{2} \lambda^{2} A^{2}\right)
$$

and hence, comparing (3.6) and (3.9), we have

$$
n<C \lambda^{2} A^{2} \frac{a_{1} a_{2}}{\log (x)}+\sqrt{\omega \theta} \frac{\lambda}{\log (x)} A+\frac{\log \left(2 c C^{\prime} a_{1} a_{2} \lambda^{2} A^{2}\right)}{\log (x)} .
$$

Write $C=\frac{\mu}{\lambda^{3} \sigma} \tilde{C}$. Then, from the definitions of $a_{1}$ and $a_{2}$, and from (3.7), necessarily

$$
C \lambda^{2} \frac{a_{1} a_{2}}{\log (x)}<\frac{\tilde{C} 6.009 \mu}{c_{1} \sigma^{2}}
$$

Since $x>n a / c$ and $n>347$, we have $\frac{\log (a+c)}{\log (x)}<1$. Combining this with (3.7) we obtain that $\frac{\lambda}{\log (x)}<c_{2} \sigma$ and, further,

$$
\frac{\log \left(2 c C^{\prime} a_{1} a_{2} \lambda^{2} A^{2}\right)}{\log (x)}<0.421 \log (A)+1.858
$$

Inequality (3.10) thus implies

$$
n<\left(\frac{\mu}{\sigma^{2} c_{1}} \tilde{C} \cdot 6.009\right) A^{2}+c_{2} \sigma \sqrt{\omega \theta} A+0.421 \log (A)+1.858
$$


Since in Lemma 2.1 we have $H \geq 1+\frac{1}{\sigma}$, necessarily $H>2.0734$, whence $\omega<4.058$ and $\theta<1.27$. Further, since $\frac{\lambda}{\sqrt{a_{1} a_{2}}}<\frac{c_{2} \sigma}{\sqrt{6.009}}$ and $\lambda\left(\frac{1}{a_{1}}+\frac{1}{a_{2}}\right)<c_{2} \sigma\left(\frac{1}{2.003}+\frac{1}{3}\right)$, we have $\tilde{C}<5.262$ if $c=1, \tilde{C}<4.853$ if $c=2$ and $\tilde{C}<4.735$ if $c=3$. By combining these estimates with (3.11), we obtain, for $a \geq 14$, that

$$
n<\left(6.009 \tilde{C} \cdot \frac{\mu}{\sigma^{2}} \frac{1}{c_{1}}\right) A^{2}+2.271 c_{2} \sigma A+0.421 \log (A)+1.858 .
$$

To remove the dependence on $a$ in this bound, we appeal to the inequalities $\log (a+c) \geq$ $\log (15)$ for $c=1, \log (a+c) \geq \log (16)$ and $a \geq 14, \log (a+c) \geq \log (21)$ for $a \geq 18$ and $c=3$ and $\log (a+c)=\log (a+3)$ for $c=3$ and $a \in\{14,16,17\}$. Hence we obtain $n \leq 347$ for $c \in\{1,2,3\}$ and $a \geq 14$, provided $h=\log \left(\frac{c_{2} n \sigma}{3}+\frac{c_{2} \sigma}{2.003}\right)+1.81$. If $h=\lambda$, inequality (3.12) actually implies a stronger bound upon $n$.

For $a \leq 13$ and $c \in\{1,2,3\}$, we omit the general estimates and use exact values for $a$. We will provide details in case $a=3$ and $c=2$; the other cases proceed in a similar fashion. We first note that direct calculation of the bounds in Lemma 2.1 with the same parameters as previously, and with $a=3, c=2, x>347 a / c$, yields an initial upper bound for $n$ of the shape $n<446$. For each prime $n$ between 347 and 446 we apply an algorithm of Pethő [35] (essentially nothing more than an analysis of convergents in the infinite simple continued fraction expansions to $\sqrt[n]{b / a}$ ) to search for solutions to our Thue inequality with $x \leq 10^{500}$. After a short computation, we find that the only such solution is $(x, y)=(1,1)$. We may thus assume that $x>10^{500}$. Using this, (3.10) now yields $n \leq 326$, as desired.

3.2. The hypergeometric method. Theorem 3.1 leaves us with only finitely many fixed exponents to treat in (3.3). In this subsection, we will assume that $n$ is either 4 or an odd prime between 3 and 347 . We first apply Lemma 2.2 to (3.3). Observe, that

$$
\max \left\{a x^{n},(a+c) y^{n}\right\} \geq a,
$$

so if

$$
a \geq \mu_{n} c^{\alpha_{n}}
$$

then (3.3) has at most one solution. Put $a_{0}(n)=\mu_{n} 3^{\alpha_{n}}$. We remark here, that $a_{0}(3)=$ $22678753, a_{0}(4)=23943$ and $a_{0}(n) \leq 1103$ for all other values of $n$. We thus need consider (3.3) only with $a \leq a_{0}(n)$. Note that (3.3) implies the inequality

$$
\left|\sqrt[n]{1+\frac{c}{a}}-\frac{x}{y}\right| \leq \frac{3}{a n y^{n}}
$$

To deduce an upper bound for $y$ in (3.3) we combine (3.13) with Lemmata 2.3, 2.4 and 2.5. We thus have 
- for $n=3$ :

$$
y<\left(\frac{12 \cdot \kappa(c) \cdot 10^{4 \lambda_{3}}}{n}\right)^{\frac{1}{n-\lambda_{3}}},
$$

- for $n \in\{4,5,7,11,13\}$ :

$$
y<\left(\frac{3 \cdot 10^{10 \lambda_{4}}}{n}\right)^{\frac{1}{n-\lambda_{4}}}
$$

- for $17 \leq n \leq 347$ :

$$
y<\left(\frac{3 C_{2}(\sqrt[m]{a+c}+\sqrt[m]{a})^{m}}{a n}\right)^{\frac{1}{n-\lambda_{1}}} .
$$

If we assume that

$$
(a, c) \notin\{(1,1),(1,2),(1,3),(2,3)\},
$$

routine computations in MAPLE show that these bounds are less then $10^{1000}$, except for some "small" values of $a$ and $n$, where we can appeal to PARI/GP to solve the corresponding Thue equations directly. By a well known theorem of Legendre, we have that in (3.3) the ratio $x / y$ is a convergent in the continued fraction expansion of $\sqrt[n]{1+\frac{c}{a}}$. We can thus apply the aforementioned algorithm of Pethô [35] to compute all solutions of the occurring inequalities. The exceptional cases here which do not satisfy the requirements of Lemmata 2.3, 2.4 and 2.5 (again, all with "small" values of $a$ and $n$ ) may also be treated via PARI/GP. It remains to deal with the pairs

$$
(a, c) \in\{(1,1),(1,2),(1,3),(2,3)\}
$$

for $n=4$ or prime $n, 3 \leq n \leq 347$. In case $(a, c)=(1,1)$, the desired result is an immediate consequence of Proposition 5.1 of [13]; we find an additional solution with $n=3$ and $(x, y)=(5,4)$. Suppose next that $(a, c)=(1,3)$. The Diophantine equations

$$
x^{n}-4 y^{n}= \pm 1, \pm 2
$$

can be shown to have no solutions in positive integers for $n \geq 3$ by combining work of Ribet [36] with elementary arguments, while

$$
x^{n}-4 y^{n}= \pm 3
$$

has no solutions in integers $x$ and $y$ with $|x y|>1$, provided $n$ has a prime divisor $p \geq 7$ (see Theorem 1.2 of [15]). It remains, therefore, to treat inequality (3.3) with $(a, c)=$ 
$(1,2)$ or $(2,3)$ and $n \in\{3,4,5,7,11,13,17\}$, and $(a, c)=(1,3), n \in\{3,4,5\}$. We appeal to PARI/GP and find no further nontrivial solutions to (3.3), unless $(a, c, n)=$ $(1,2,3)$ (where there is the additional solution $(x, y)=(3,2))$ or $(a, c, n)=(2,3,3)$ (where we have $(x, y)=(19,14)$ ). This completes the proof of Case I.

Case II can be handled similarly. We can assume, for the remainder of the proof, that for any positive solution $(x, y)$ of $(3.1)$, we have $x \geq 2$. Denote by $\left(x_{0}, y_{0}\right)$ a known solution of (3.1). As previously, we may conclude from Lemma 2.2 that if $\max \left(x_{0}, y_{0}\right)$ is larger than a computable constant $X_{n}$, then the only positive solution of $(3.1)$ is $\left(x_{0}, y_{0}\right)$. Hence, we have only to consider (3.1) with $n \in\{3,4,5\}$ and with a given finite set $\mathcal{X}$ of the pairs $\left(x_{0}, y_{0}\right)$. By way of example, if $a=1$ and $n=3$, we have $2 \leq x_{0} \leq 283$, and determine $b y_{0}^{3}$ by factoring $a x_{0}^{3}+t$ for $t \in\{ \pm 1, \pm 2, \pm 3\}$. In general, applying Lemma 2.2 to our set of pairs $\mathcal{X}$, we arrive at a finite set of possible pairs $(a, b)$, with corresponding finite set of Thue inequalities (really, in this case, equations) to solve. In most cases, we can carry this out easily via the hypergeometric method. Assume that $\left(x_{0}, y_{0}\right)$ is given and that $a x_{0}^{n}-b y_{0}^{n}=-t$, with $t \in\{ \pm 1, \pm 2, \pm 3\}$. Then $b$ can be written as $\frac{a x_{0}^{n}+t}{y_{0}^{n}}$ and, after substituting this into (3.1), we find that

$$
\left|a x^{n}-\frac{a x_{0}^{n}+t}{y_{0}^{n}} y^{n}\right| \leq 3 .
$$

Applying Lemmata 2.4 and 2.5, we are led to inequalities of the shape

$$
\frac{c_{1}}{\left(x_{0} y\right)^{\lambda}}<\left|\frac{x y_{0}}{x_{0} y}-\sqrt[n]{1+\frac{t}{a x_{0}^{n}}}\right| \leq \frac{3 \cdot y_{0}^{n}}{a\left(x_{0} y\right)^{n}},
$$

where the constant $c_{1}$ can be deduced from the statements of Lemmata 2.4 or 2.5. This yields, in a similar fashion to Case I, that $y$ is bounded by some absolute constant (usually around $\left.10^{500}\right)$. From (3.1),

$$
\left|\frac{x}{y}-\sqrt[n]{\frac{b}{a}}\right|<\frac{3}{a n y^{n}}
$$

and hence, via Legendre's theorem, we have that $x / y$ is a convergent in the simple continued fraction expansion of $\sqrt[n]{b / a}$. Thus, we may again apply Pethő's algorithm [35] to compute all solutions of the corresponding inequalities. Repeating this procedure for all $\left(x_{0}, y_{0}\right) \in \mathcal{X}$, and using PARI/GP for some exceptional equations with small coefficients which we are unable to handle via the hypergeometric method, we conclude that (3.1) has at most one solution for each triple $(a, b, n)$ in Case II. This completes the proof of Theorem 1.4. Full details of these computations are available from the authors upon request. 


\section{Concluding remarks}

Due to the limitations of the hypergeometric method and lower bounds for linear forms in logarithms, it was necessary for us to solve a number of Thue equations of relatively high degree (up to 31). We would like to express our thanks to Guillaume Hanrot who wrote an extension of PARI which contains a new treatment of Thue equations based on his paper [27]. In this paper, he showed that the knowledge of a subgroup of finite index in the unit group is actually sufficient to solve Thue equations. With this software we were able to solve Thue equations of quite high degree in a reasonable amount of time and obtain a result independent of the Generalized Riemann Hypothesis.

It is worth noting that extremely careful application of the techniques of [10] would enable one to replace the upper bound of $n \leq 347$ in the definition of the exceptional set $T$ by $n \leq 53$. To carry this out would be of practical interest only in the event that the remaining lower degree Thue equations could be explicitly solved without dependence upon the GRH to certify the putative fundamental units in the number fields encountered.

As a final note, the first author would like to acknowledge that Theorem 2.1 of [12], which claims that (in the notation of the current paper) $S_{2}$ is empty, overlooks the family of equations of the shape $x^{n}-3 y^{n}=2$ which contribute to our set $T_{3}$. This mistake was due to an incorrect conductor calculation of a corresponding Frey curve. The first author regrets any confusion caused by this.

\section{Acknowledgements}

We would like to express our thanks to Kálmán Gyôry, Ákos Pintér and Attila Bérczes for their helpful comments, and also to László Kovács for providing us with the necessary equipment for our numerical computations.

\section{References}

[1] A. BAKer, Rational approximations to certain algebraic numbers, Proc. London Math. Soc. (3) 14 (1964), 385-398.

[2] A. BAKER, Rational approximations to $\sqrt[3]{2}$ and other algebraic numbers, Quart. J. Math. Oxford Ser. (2) 15 (1964), 375-383.

[3] A. BAKER, Simultaneous rational approximations to certain algebraic numbers, Proc. Cambridge Phil. Soc 63 (1967), 693-702.

[4] A. BAKER, Contributions to the theory of Diophantine equations, Phil. Trans. Roy. Soc. London, 263 (1968), 173-208.

[5] A. BAZsó, A. BÉrcZes, K. GyôRY and Á. PinTÉr, On the resolution of equations $A x^{n}-B y^{n}=C$ in integers $x, y$ and $n \geq 3$, II, Publ. Math. Debrecen 76 2010, 227-250. 
[6] A. BAZsó, On binomial Thue equations and ternary equations with S-unit coefficients, Publ. Math. Debrecen 77 (2010), 499-516.

[7] M. A. BennetT, Simultaneous rational approximation to binomial functions, J. Austral. Math. Soc. 348 (1996), 1717-1738.

[8] M. A. BennetT, Effective measures of irrationality for certain algebraic numbers, J. Austral. Math. Soc. 62 (1997), 329-344.

[9] M. A. BENNETT, Explicit lower bounds for rational approximation to algebraic numbers, Proc. London Math. Soc. 75 (1997), 63-78.

[10] M. A. BEnNetT, Rational approximation to algebraic numbers of small height: the Diophantine equation $\left|a x^{n}-b y^{n}\right|=1$, J. Reine Angew. Math. 535 (2001), 1-49.

[11] M. A. Bennett, Products of consecutive integers, Bull. London Math. Soc. 36 (2004), 683-694.

[12] M. A. Bennett, Powers in recurrence sequences : Pell equations, Trans. Amer. Math. Soc. 357 (2004), 1675-1691.

[13] M. A. Bennett, The Diophantine equation $\left(x^{k}-1\right)\left(y^{k}-1\right)=\left(z^{k}-1\right)^{t}$, Indag. Math. (N.S.) 18/4 (2007), 507-525.

[14] M. A. Bennett, K. GyőRy, M. Mignotte and Á. PIntÉr, Binomial Thue equations and polynomial powers, Compos. Math. 142 (2006), 1103-1121.

[15] M. A. BENNETT and C. SKInNER, Ternary Diophantine equations via Galois representations and modular forms, Canad. J. Math. 56 (2004), 23-54.

[16] M. A. Bennett and B. M. M. DE Weger, On the Diophantine equation $\left|a x^{n}-b y^{n}\right|=1$, Math. Comp. 67 (1998), 413-438.

[17] A. BÉRCZES and A. PETHŐ, On norm form equations with solutions forming arithmetic progressions, Publ. Math. Debrecen 65 /3-4 (2004), 281-290.

[18] A. BÉRCZES and A. PETHŐ, Computational experiences on norm form equations with solutions from an arithmetic progression, Glas. Mat. Ser. III 41(61) (2006), 1-8.

[19] E. Bombieri and W. Schmidt, On Thue's equation, Invent. Math. 88 (1987), 69-81.

[20] G. V. Chudnovsky, On the method of Thue-Siegel, Ann. of Math. (2) 117 (1983), 325-382.

[21] J.H. EVERTSE, Upper Bounds for the numbers of solutions of diophantine equations, PhD Thesis, Leiden, 1983.

[22] K. GYŐRY, L. HAJdU and N. SARADHA, On the Diophantine equation $n(n+d) \cdots(n+(k-1) d=$ $\left.b y^{l}\right)$, Canad. Math. Bull. 47 (2004), 373-388.

[23] K. GYőRY, I. PINK and Á. PINTÉR, Power values of polynomials and binomial Thue-Mahler equations, Publ. Math. Debrecen 65 (2004), 341-362.

[24] K. GYôRY and Á. PINTÉR, Almost perfect powers in products of consecutive integers, Monatsh. Math. 145 (2005), 19-33.

[25] K. GYôRY and Á. PINTÉR, On the resolution of equations $A x^{n}-B y^{n}=C$ in integers $x$, y and $n \geq 3$, I, Publ. Math. Debrecen 70 (2007), 483-501.

[26] K. GYŐRY and Á. PINTÉR, Binomial Thue equations, ternary equations and power values of polynomials (in Russian), Fundam. Prikl. Mat. 16 /5 (2010), 61-77.

[27] G. HANROT, Solving Thue equations without the full unit group, Math. of Comp. 69, no. 229, (1997), 395-405.

[28] M. LAURENT, Linear forms in two logarithms and interpolation determinants II, Acta Arith. 133/4 (2008), 325-348.

[29] W. LJUngGRen, On an improvement of a theorem of T. Nagell concerning the Diophantine equation $A x^{3}+B y^{3}=C$, Math. Scand. 1 (1953), 297-309. 
[30] K. MAHLER, Ein Beweis des Thue-Siegelschen Satzes über die Approximation algebraischer Zahlen für binomische Gleichungen, Math. Ann. 105 (1931), 267-276.

[31] K. MAHLER, Zur Approximation algebraischer Zahlen, I: Über den grössten Primteiler binärer Formen, Math. Ann. 107 (1933), 691-730.

[32] M. Mignotte, A note on the equation ax ${ }^{n}-b y^{n}=c$, Acta Arith. 75 (1996), 287-295.

[33] J. MUeller and W. SChMidt, Thue's equations and a conjecture of Siegel, Acta Math. 160 (1988), 207-247.

[34] PARI/GP, version 2.3 . 4, Bordeaux, 2011, http://pari.math.u-bordeaux.fr/.

[35] A. РетHô, On the resolution of Thue inequalities, J. Symbolic Comput. 4 (1987), 103-109.

[36] K. A. RiBET, On the equation $a^{p}+2^{\alpha} b^{p}+c^{p}=0$, Acta Arith. 79 (1997), 7-16.

[37] C. L. SiEgEL, Die Gleichung $a x^{n}-b y^{n}=c$, Math. Ann. 114 (1937), 57-68.

[38] A. Thue, Über Annäherungswerte algebraischer Zahlen, J. Reine Angew. Math. 135 (1909), 284-305.

[39] R. Tijdeman, Some applications of Baker's sharpened bounds to Diophantine equations, Séminaire Delange-Pisot-Poitou (16e année: 1974/75), Théorie des nombres, Fasc. 2, Exp. No. 24, Secrétariat Mathématique, Paris, 1975, 7.

\author{
DEPARTMENT OF MATHEMATICS \\ UNIVERSITY OF BRITISH COLUMBIA \\ VANCOUVER \\ BRITISH COLUMBIA \\ V6T $1 Z 2$ \\ CANADA \\ E-mail: bennett@math.ubc.ca \\ INSTITUTE OF MATHEMATICS \\ UNIVERSITY OF DEBRECEN \\ H-4010 DEBRECEN, P.O. BOX 12, HUNGARY \\ E-mail: pinki@science.unideb.hu
}

MTA-DE RESEARCH GROUP "EQUATIONS FUNCTIONS AND CURVES"

HUNGARIAN ACADEMY OF SCIENCES AND UNIVERSITY OF DEBRECEN

H-4010 DEBRECEN, P.O. BOX 12, HUNGARY

E-mail: zsrabai@science.unideb.hu 